Implementing Graph Pattern Queries on a Relational Database

I. L. Kaplan, G. M. Abdulla, S. T. Brugger, S. R. Kohn

January 8, 2008 
This document was prepared as an account of work sponsored by an agency of the United States government. Neither the United States government nor Lawrence Livermore National Security, LLC, nor any of their employees makes any warranty, expressed or implied, or assumes any legal liability or responsibility for the accuracy, completeness, or usefulness of any information, apparatus, product, or process disclosed, or represents that its use would not infringe privately owned rights. Reference herein to any specific commercial product, process, or service by trade name, trademark, manufacturer, or otherwise does not necessarily constitute or imply its endorsement, recommendation, or favoring by the United States government or Lawrence Livermore National Security, LLC. The views and opinions of authors expressed herein do not necessarily state or reflect those of the United States government or Lawrence Livermore National Security, LLC, and shall not be used for advertising or product endorsement purposes.

This work performed under the auspices of the U.S. Department of Energy by Lawrence Livermore National Laboratory under Contract DE-AC52-07NA27344. 


\section{Graph Query Language : Implementing Graph Pattern Queries on a Relational Database}

This page last changed on Jan 11, 2008 by kaplan4.

LLNL technical report LLNL-TR-400310. This work performed under the auspices of the U.S. Department of Energy by Lawrence Livermore National Laboratory under Contract DE-AC52-07NA27344. Funding provided by LDRD 06-ERD-009.

\section{Abstract}

When a graph database is implemented on top of a relational database, queries in the graph query language are translated into relational SQL queries. Graph pattern queries are an important feature of a graph query language. Translating graph pattern queries into single SQL statements results in very poor query performance. By taking into account the pattern query structure and generating multiple SQL statements, pattern query performance can be dramatically improved. The performance problems encountered with the single SQL statements generated for pattern queries reflects a problem in the SQL query planner and optimizer. Addressing this problem would allow relational databases to better support semantic graph databases. Relational database systems that provide good support for graph databases may also be more flexible platforms for data warehouses.

\section{Introduction}

A semantic graph database is a database made up of typed graph vertices and typed edges that connected these vertices. For example, a graph database could include vertices with the type person or organization. An example of a person vertex might be \{name=Thomas $\mathrm{J}$. Watson, birthdate = 1914-01-14 \}. This instance of a person vertex in the graph could be connected by an EmployedBy edge to the organization vertex International Business Machines and a BornIn edge to the city Dayton, ohio. As this example demonstrates, a vertex in the graph database may have multiple attributes (e.g., name and birth date). Edges may, optionally, have attribute values as well.

A graph query language is a query language designed for a graph database. When a graph database is implemented on top of a relational database, queries in the graph query language are translated into relational SQL queries [1]. Some graph query operations can be efficiently implemented by translating the graph query into a single SQL statement. Examples include adjacency and graph filter queries. The adjacency function adds a set of selected adjacent links and vertices to a selected subgraph. The graph filter function filters a graph to return a subgraph consisting of only selected vertices or links. Graph pattern queries are an important feature of a graph query language. In contrast to the adjacency and graph filter functions, translating pattern queries into single SQL statements can result in very poor query performance.

In this report, the performance of single SQL statement pattern queries is contrasted with an approach that generates SQL for depth first paths through the pattern query. The performance of this latter approach can result in dramatically faster graph pattern query execution. Pattern queries that are translated into SQL for depth first paths will also perform better as the graph size increases.

Generating SQL for paths through the pattern query requires more complex software support in the graph 
query engine. This extra complexity could be avoided if the relational SQL optimizer and query planner generated an efficient query plan for single SQL statements generated for pattern queries. The fact that this is not the case represents a failure of the SQL query optimizer, relative to graph pattern queries.

\section{Graph Databases and Graph Queries}

In some application areas, graph databases offer important advantages over relational databases:

1. Data from a wide variety of sources can be easily fused into a single semantic graph.

2. The structure of the graph is controlled by the semantic graph ontology. The graph structure can be changed by modifying the ontology. Within limits, software can migrate graph data from one graph ontology to another.

3. Graph queries provide a simple way to express graph database queries would be difficult to express in relational SQL.

Many graph pattern matching algorithms [6] are implemented as in memory algorithms. In most cases these algorithms can only be applied to data sets that will fit in computer memory. A semantic graph database can store data from myriad sources, resulting in very large graphs that cannot fit into computer memory. The pattern matching algorithms that are applied to out-of-memory graphs stored on persistent media like computer disk systems are data parallel algorithms. In this report these algorithms are implemented in relational SQL.

A graph database could be supported by custom database software. A graph query would be directly translated into a query plan for this custom database. Such a database would require significant development effort and might have to be modified for different hardware configurations. Translating graph queries into standard SQL for a relational database allows the graph database to be hosted on a variety of relational database systems, ranging from database servers to distributed relational databases.

The graph query language discussed here [5] supports the following graph functions:

- graph union, intersection and difference

- filter - filter a graph so that the query result contains only the vertices and edges defined in the query.

- adjacency - expand a defined subgraph by adding selected links and vertices.

- Path traversal between a set of source and destination points in the graph.

- Pattern queries.

The graph query language supports nested queries, where one query expression defines the input graph for another query expression.

The graph query language described here has been influenced by the work of David Jensen's group at the University of Massachusetts at Amherst on the QGraph graph query language $[2,3]$ and by the work that David Silberberg's group has done at the Johns Hopkins Advanced Physics Laboratory [4].

The binary graph operations (union, intersection and difference), filter and adjacency are implemented by translating the graph query into a single SQL statement. This approach is attractive because of its simplicity. A similar approach can be used for graph pattern queries. The SQL for a pattern query could also be constructed by traversing the data structure for the pattern query. As the results presented in this report show, when a single SQL statement is generated for a graph pattern query, the query performance 
can be very poor.

\section{Graph Database Ontologies}

A graph database is created when a graph ontology is loaded into the semantic graph database system. The graph ontology defines the graph vertex types and the types of the edges that connect these vertices. The ontology also defines the attributes for each vertex (or edge). Multiple ontologies can be supported at the same time and there may be multiple graphs associated with each ontology.

The graph database schema that supports the graph ontologies is diagrammed in Figure 1, below. Each ontology has an associated set of tables that store the vertex attributes. In Figure 1 the table es_42 stores attribute information for instances of author vertices and the table es_43 stores the attributes for instances of article vertices. The cloud represents the ontology information which includes mappings between the names in the query (author or article) and database tables (es_42 or es_43). Graph vertex attributes are shared by all graphs that are associated with a particular ontology. There is a unique edge table for each graph. The edge table columns are also diagrammed in Figure 1. Each graph also has an associated vertex table that contains all of the vertex identifiers and types for the graph. This table is not used by pattern query operations.

\section{Graph Database Schema}

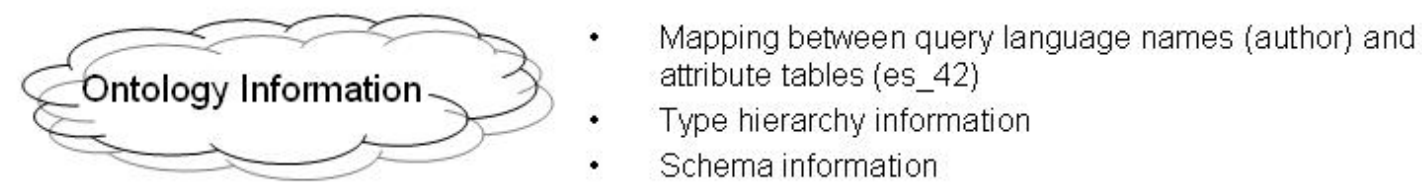

\begin{tabular}{|l|l|l|l|l|l|}
\hline src_id & src_type & edge_id & edge_type & dst_id & dst_type \\
\hline
\end{tabular}

Table es_42

\begin{tabular}{|l|l|l|l|l|}
\hline node_id & LastName & FirstName & suffix
\end{tabular} $2 \begin{gathered}\text { Author } \\
\text { LastName: string } \\
\text { FirstName: string } \\
\text { Suffix: string }\end{gathered}$

Table es_43

\begin{tabular}{|l|l|l|l|l|}
\hline node_id & PMID & title & abstract & Language \\
\hline
\end{tabular}

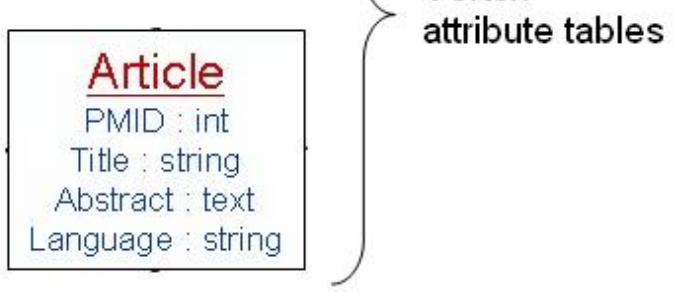

Figure 1

The SQL generated for the pattern queries discussed in this report largely consist of self-joins against the edge table. The edge table has three sets of indices, as shown in Figure 2, below. The primary key is on the $\{$ link_id, link_type\} columns. There are also indices on the \{src_node_id, src_node_type and the \{dst_node_id, dst_node_type\} columns. 


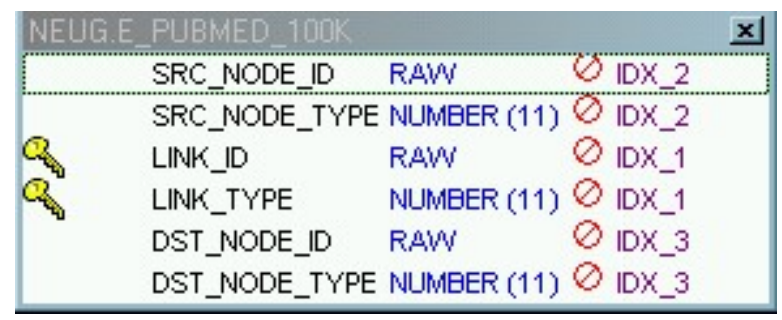

Figure 2

\section{Pattern Queries}

"I don't know what you mean by 'pattern query'," Alice said.

Humpty Dumpty smiled contemptuously. "Of course you don't - till I tell you. When I say find all of the authors who have published two articles, I mean return a set of subgraphs that pair each author with combinations of two of the articles they have published. It's a combinatoric choose $(\mathrm{N}, \mathrm{k})$ problem you see."

"But a pattern query doesn't mean 'choose $(\mathrm{N}, \mathrm{k})$ ', it just means find that pattern in the graph" Alice objected.

"When I use a word," Humpty Dumpty said in a rather a scornful tone, "it means just what I choose it to mean - neither more nor less.

"The question is," said Alice, "whether you can make words mean different things."

With apologies to Lewis Carroll and his book Through the Looking-Glass (Macmillan, 1871)

Pattern queries seem intuitive. For example, the pattern query find all of the authors who have published two articles seems intuitively obvious. But what does this query really mean? Does it mean find all of the authors who have published exactly two articles? Or does it mean find all of the authors who have published two or more articles? If we use the latter meaning, what subgraphs should the query return?

The pattern query find all of the authors who have published two articles is constrained only by the types of the vertices (or the edges if we defined the edge relationship in the pattern query). As Humpty Dumpty states in the quote above, computational complexity of the pattern query find all authors who have published two articles is choose $(N, 2)$, where $N$ is the number of articles published by a particular author. This is shown in Figure 3, below. The author Peter Watts has four publications: Starfish, Maelstrom, Behemoth and Blindsight. 


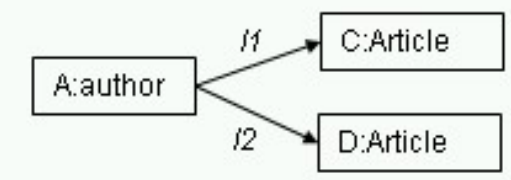

Pattern query

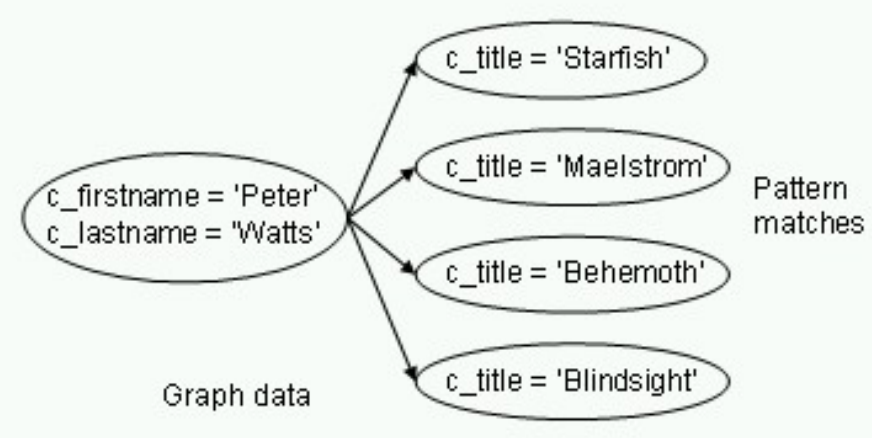

Figure 3

The number of unique patterns in this case is $(3 * 4) / 2=6$. The number of subgraphs that are returned by choose $(\mathrm{N}, \mathrm{k})$ pattern queries can be calculated using the equation below:

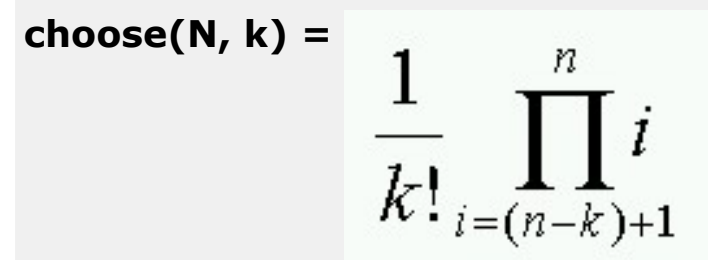

\section{Why Allow Combinatoric Pattern Queries?}

The behavior of pattern queries that are constrained only by the vertex type may be unexpected. The person posing the query may not expect to get back a set of subgraphs consisting of the combinatoric set of $\operatorname{choose}(N, k)$ matching subgraphs. Why allow queries that return a large unexpected result?

In some cases combinatoric pattern matching is the only technique that will find a matching pattern in the graph. In Figure 4, below, the pattern query (shown with rectangles) matches only one pattern in the data (shown with ovals). This pattern can only be discovered by trying the different combinations to fill in the matching subgraph. 


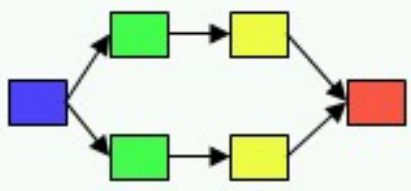

Pattern Query

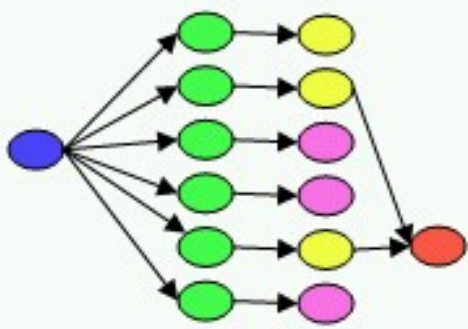

Graph Data

\section{Figure 4}

Combinatoric matches are only a problem when there are instances of high degree vertices for one of the types in the pattern. If the pattern consists of types with only a relatively small number of instances the combinatoric match may not be a problem.

\section{Queries with Constraints}

By adding constraints to a pattern query, the number of subgraphs returned can be reduced. There are two kinds of constraints:

1. a where clause that defines a constraint on the vertex attributes

2. a cardinality constraint that defines an exact number of edges.

These constraints can be mixed in a single pattern query.

An example of a query with where clause constraints is shown below in Figure $\mathbf{5}$. 


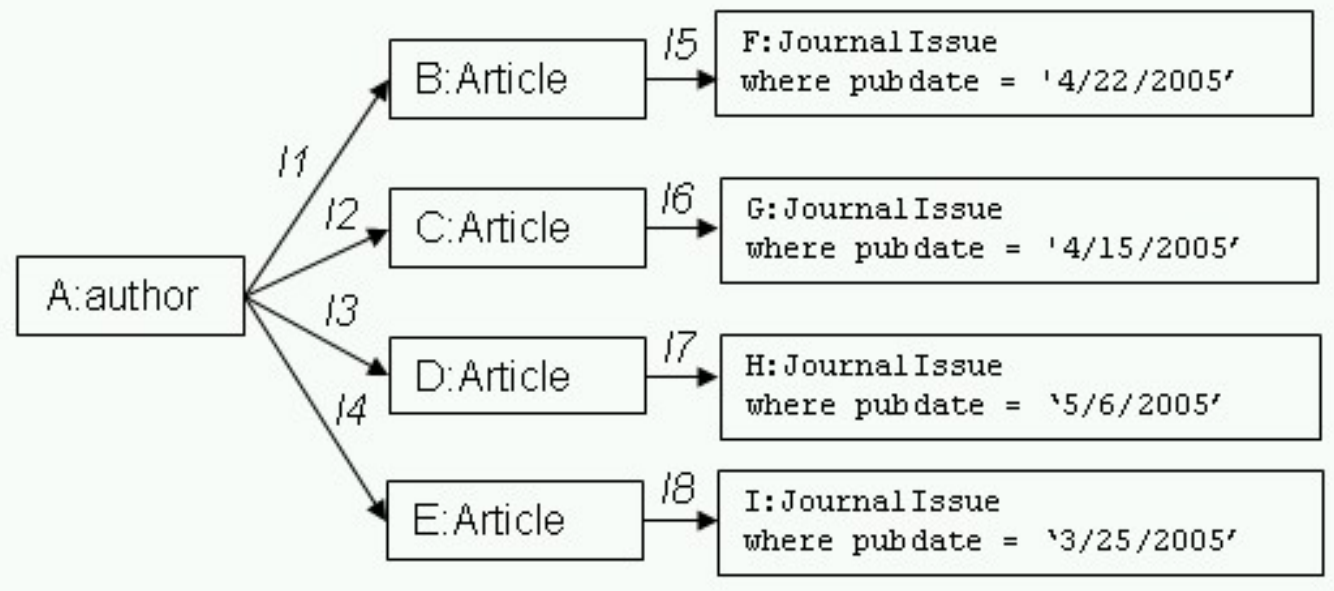

\section{Figure 5}

The pattern query in Figure 6 includes a cardinality constraint. This query finds all of the authors who have published exactly two articles.

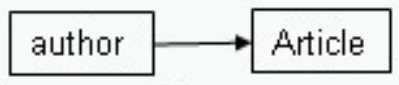

[2]

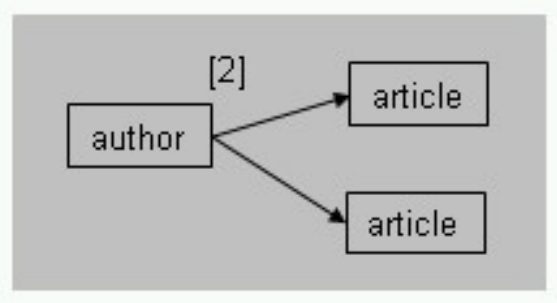

\section{Figure 6}

A cardinality constraint may also specify a range. Figure $\mathbf{7}$ shows a query that will return subgraphs for authors who have published exactly two, three or four articles.

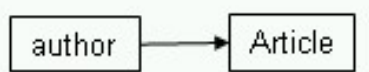

$[2 \ldots 4]$

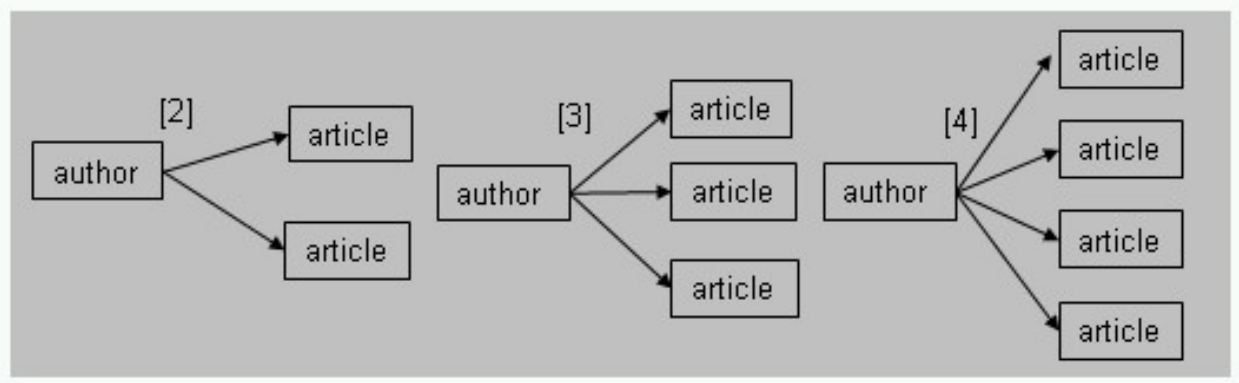

\section{Figure 7}

A cardinality constraint may have an open upper bound. The query in Figure 8 will return subgraphs for authors have have published 2 to $\mathrm{N}$ articles, where $\mathrm{N}$ is the maximum number of articles published by any author. 


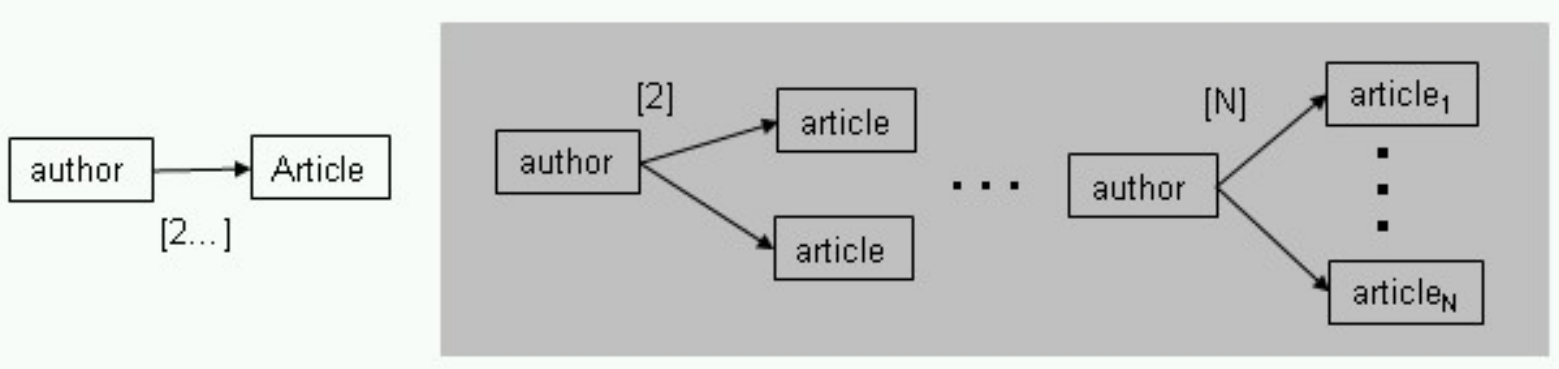

Figure 8

\section{Pattern Query to SQL Translation}

A graph pattern query is a connected graph. Execution of a pattern query is the process of finding subgraphs that exactly match the graph defined in the pattern query.

A graph pattern query is stored as a data structure in computer memory. The query is translated into SQL by traversing this data structure and building one or more SQL statements which are sent to the relational database to produce the pattern query result. The simplest way to translate pattern queries into SQL is to generate a single SQL statement, with an edge table join for each edge in the graph. Unfortunately on the database we used for the benchmarks in this report, the performance of these queries is poor. By traversing unique non-overlapping paths through the pattern query and generating SQL for each path, much faster pattern query execution can be achieved.

\section{Avoiding Geometric Pattern Duplicates}

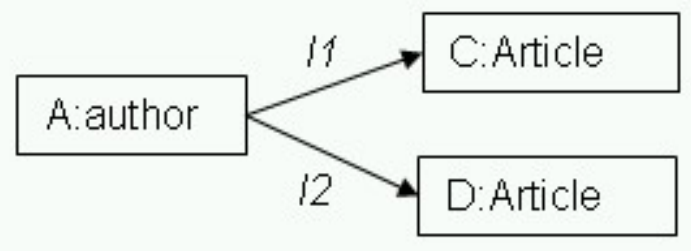

\section{Figure 9}

There is no inherent ordering of vertices in a graph. Figure $\mathbf{1 0}$ shows two possible orderings for one subgraph returned by the pattern query in Figure $\mathbf{9}$ (an author linked to two articles). 


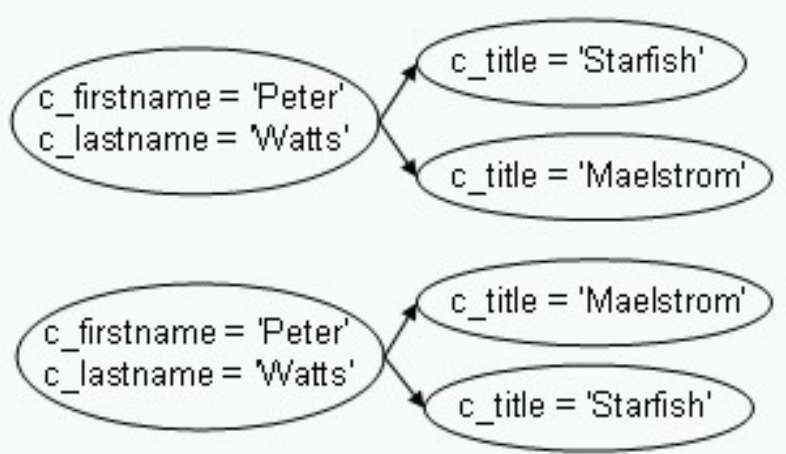

Figure 10

Patterns that differ only in their geometric arrangement are of no interest in graph pattern matching applications and greatly increase the size of the query result. To avoid returning these geometric duplicates the SQL that is generated for a pattern query forces an ordering to eliminate duplicate patterns. An example is shown below in the SQL that would be generated for the author to two articles query.

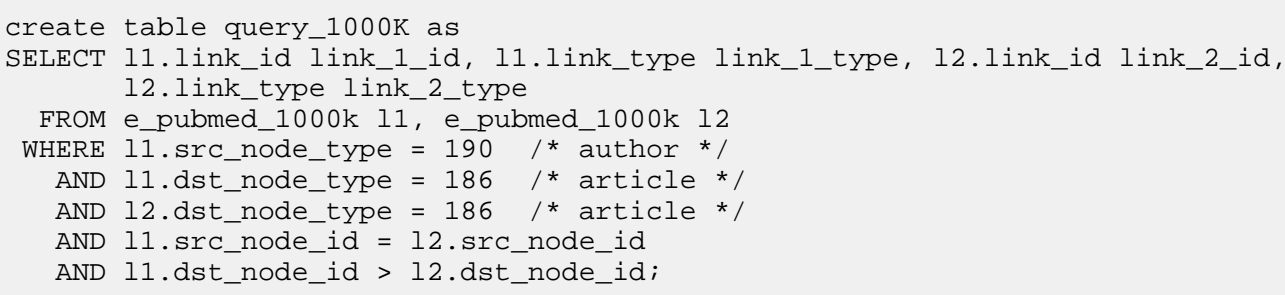

The source and destination node (or vertex) ID is an integer or a cryptographic hash value that is globally unique for an ontology (e.g., no other node in a graph associated with that ontology has that ID value). The greater than constraint on the destination of the two author to article edges forces an ordering for the article vertices in the matching subgraphs. This eliminates geometric duplicates.

\section{The Pattern Query Result Table}

The result of a pattern query is the unique set of subgraphs that match the graph pattern. The SQL for pattern queries in this report return a result where each matching subgraph is a row in the result table. Each column of the row is a link in the matching subgraph. This presents a problem since pattern query result tables may have differing numbers of result table columns. The query engine will execute additional queries that write the query result into a query result table that has the same column structure for all pattern queries. These queries are outside of the scope of this report.

\section{The Relational Database and Test Graph Size}

All of the benchmarks discussed in this report have been run on a relational database that is hosted on a four processor database server. The graph used for benchmark testing consists of 1,002,970 vertices (i.e., about 1 million vertices) and 1,929,797 edges (i.e., about 2 million edges). The important metric here is the number of edges (e.g., the edge table size). The graph data used for these benchmarks is taken from the PubMed data set, which consists of journal article publication information. This graph is 
considerably smaller than the graphs that would be seen in actual semantic graph applications.

\section{Pattern Queries with Cardinality Constraints}

The pattern query in Figure $\mathbf{1 1}$ includes a cardinality constraint. This pattern query will find all of the authors who have published exactly two articles.

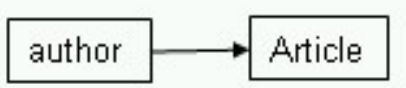

[2]

\section{Figure 11}

The SQL for a pattern query with a cardinality constraint requires nested SQL that finds the author vertices for the authors who have published exactly two articles. This nested SQL is shown below:

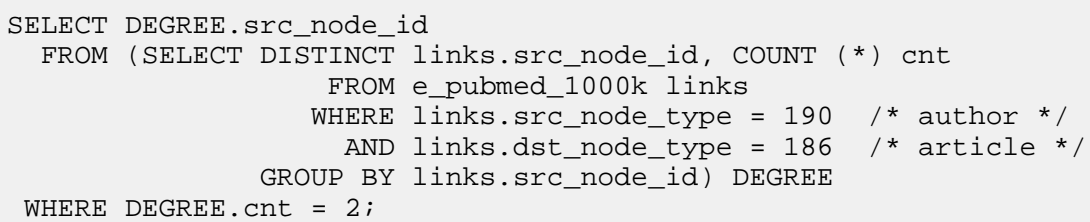

This SQL query returns only the author vertices, not the complete pattern. In order to return the complete pattern, this query is included as a subquery in a larger SQL query. This is shown below:

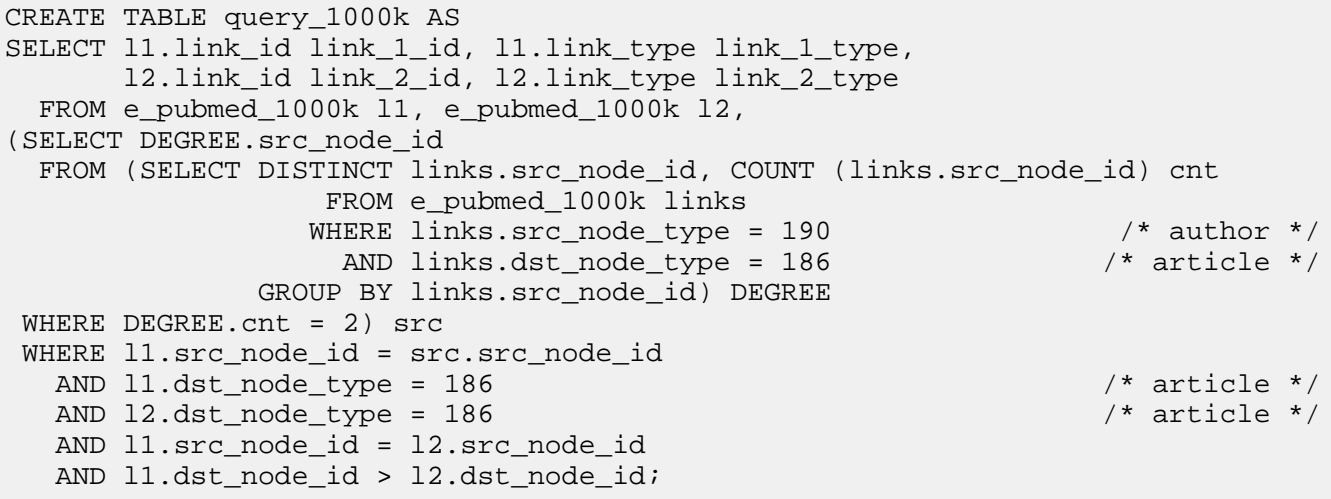

The above SQL statement, with the nested query to find author vertex cardinality caused problems for the query optimizer and query planner, resulting in poor query performance on the database we used for our tests. The performance of this query on the test graph is shown below:

\begin{tabular}{|c|c|}
\hline \multicolumn{1}{|c|}{ time (seconds) } & \multicolumn{1}{c|}{ rows } \\
\hline 8640 (2.4 hours) & 66497 \\
\hline
\end{tabular}

The fact that this query has such poor performance seems to be an aberration of the database we used for testing (perhaps a bug). Another relational database we used for testing executed this query in about 
the same time as the query broken into two steps, discussed below.

To avoid this problem, this query was broken up into two steps:

1. Build a table of author vertices that are linked to two articles.

2. Return the author to article pattern that contains these articles.

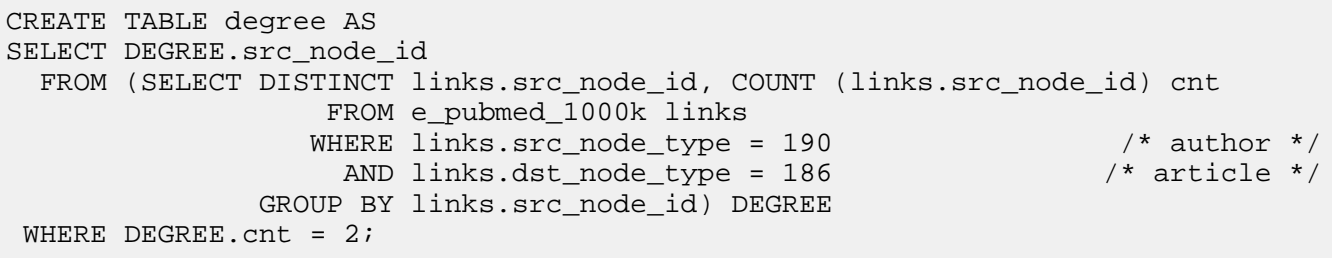

\begin{tabular}{|c|c|c|}
\hline & time (seconds) & rows \\
\hline Step 1 & 5.2 & 66497 \\
\hline Step 2 & 2.8 & 66497 \\
\hline Total time & 8.0 & \\
\hline
\end{tabular}

\section{Optimizing Graph Pattern Query Execution}

One of the simplest ways to implement graph pattern queries on top of a relational database is to translate a pattern query into a single SQL statement. The pattern query translation software traverses the pattern query data structure and builds an SQL statement for the pattern query. As the benchmarks below demonstrate, these SQL queries consist of multiple self-joins against the graph edge table.

Unfortunately the SQL optimizer and query planner does not execute these SQL statements efficiently. As the benchmarks below demonstrate, the poor execution performance for these SQL statements is not a result of the SQL statement complexity, as might be the case if there were multiple levels of query nesting. The problem seems to arise from the nature of graph pattern queries. Different edge table join orderings can produce intermediate query results that differ by orders of magnitude in size. Figure 12, below, shows a breath first and a depth first join ordering. Each color in the diagram is intended to represent a set of edge table joins. If the breath first ordering is used there would be a very large query intermediate. If a depth first join ordering is used, the query intermediate is orders of magnitude smaller resulting in dramatically faster query execution times. 


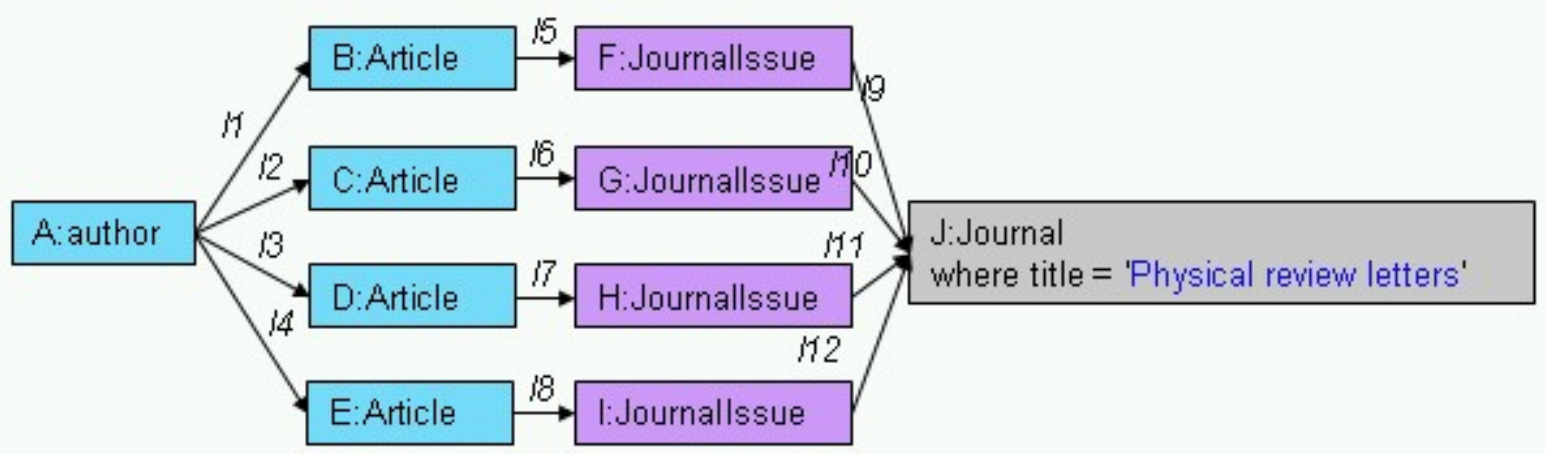

Breath First Pattern Execution

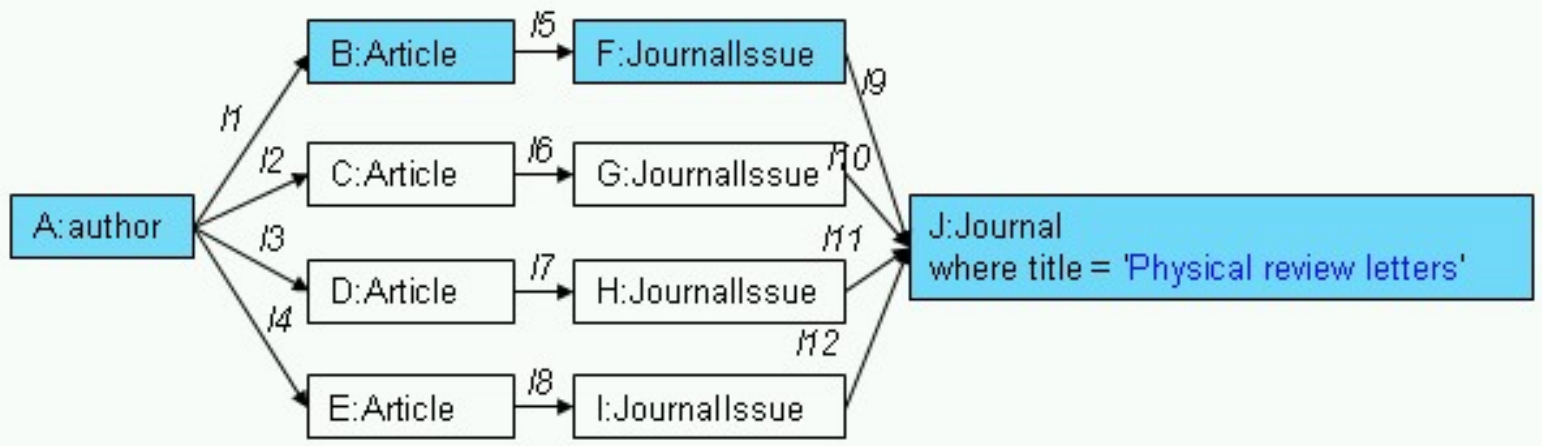

Depth First Pattern Execution

\section{Figure 12}

The benchmarks in this report compare pattern queries implemented by single SQL statements with patterns implemented by SQL generated for depth first paths through the pattern query. As these benchmarks demonstrate, multiple SQL statements that take the pattern structure into account yield much better pattern query performance than single SQL statements.

\begin{tabular}{|l|l|l|l|}
\hline \multicolumn{1}{|c|}{ query } & $\begin{array}{c}\text { number of subgraphs } \\
\text { (rows) }\end{array}$ & $\begin{array}{c}\text { single SQL statement } \\
\text { (seconds) }\end{array}$ & $\begin{array}{c}\text { paths through the } \\
\text { pattern (seconds) }\end{array}$ \\
\hline Where constraint & 1 & $8640(2.4$ hours) & $\sim 3.0$ \\
\hline Query 1 & 39036649 & 54000 (15 hours) & $1014(\sim 17$ minutes) \\
\hline Query 2 & 957272 & $\begin{array}{l}16 \text { hours (did not } \\
\text { complete) }\end{array}$ & 21.8 \\
\hline Query 3 & 19067116 & 3480.0 (58 minutes) & $387(6.45$ minutes $)$ \\
\hline Query 4 & 7630 & 6840.0 (1.9 hours) & 282.0 (4.7 minutes) \\
\hline
\end{tabular}

\section{Discussion}

Translating graph pattern queries into relational SQL that has acceptable performance can be a difficult problem. The benchmarks results described in this report were obtained for a small graph of only 1 million vertices and 2 million edges. The graphs used in real semantic graph applications would be much larger. These larger graphs may require more powerful databases systems than the database server used in these benchmarks. But hardware alone will not deliver pattern query performance. Pattern query 
execution must minimize the size of query intermediate results. The SQL optimizer cannot be relied on to efficiently execute single SQL statements that implement pattern queries. The results in this report show that acceptable pattern query execution performance requires SQL generation that optimizes for the pattern query structure.

The extra complexity entailed in translating graph pattern queries into multiple SQL statements is required because the relational database query planner and optimizer does very poorly with the single SQL statements generated for pattern queries. Graph pattern query translation must take into account the structure of the pattern query and generate multiple SQL statements to efficiently implement the pattern query. This complexity could be avoided if the relational database query optimizer were extended to handle pattern queries efficiently. One approach that the SQL optimizer might take is to rebuild the pattern query from the set of joins defined in a single SQL statement for the pattern. Once the pattern is reconstructed, the query optimizer could use a query plan that ordered joins using depth first paths through the pattern, as described in this report.

Graph databases and graph query languages allow queries to be easily formulated that are difficult to express in relational SQL. These queries are commonly used in applications involving crime or intelligence investigation. These are specialized areas and it could be argued that the effort to extend the query optimizer for graph pattern queries would not benefit a majority of the database user community. However, the problems encountered for pattern queries may also exist for data warehouse applications. The database schema chosen for many data warehouses may be overly constrained to avoid the kinds of performance problems discussed in this report. The result is a data warehouse that is tuned for certain queries but performs very badly for others. An SQL optimizer that could deliver acceptable performance for pattern queries might allow more general data warehouse schemas. The result would be a more flexible data warehouse implementation.

\section{Pattern Query Benchmark Results}

\section{A Pattern Query with a Where Clause Constraint}

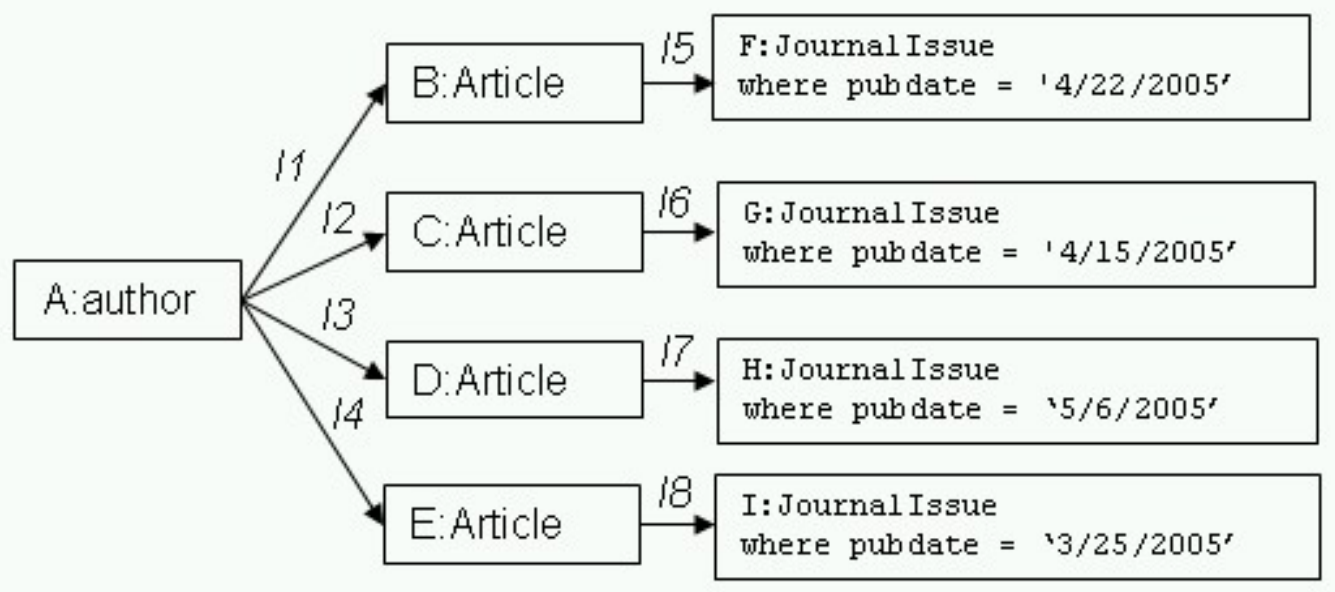

This is a highly constrained pattern query. There is only one instance of this sub-graph in the test graph. The simplest way to generate SQL for this query is to traverse the data structure for the pattern query 
and generate a single SQL statement. The SQL statement that might be generated is shown below. The es_194 table is the vertex table that contains the attribute values for the JournalIssue vertices.

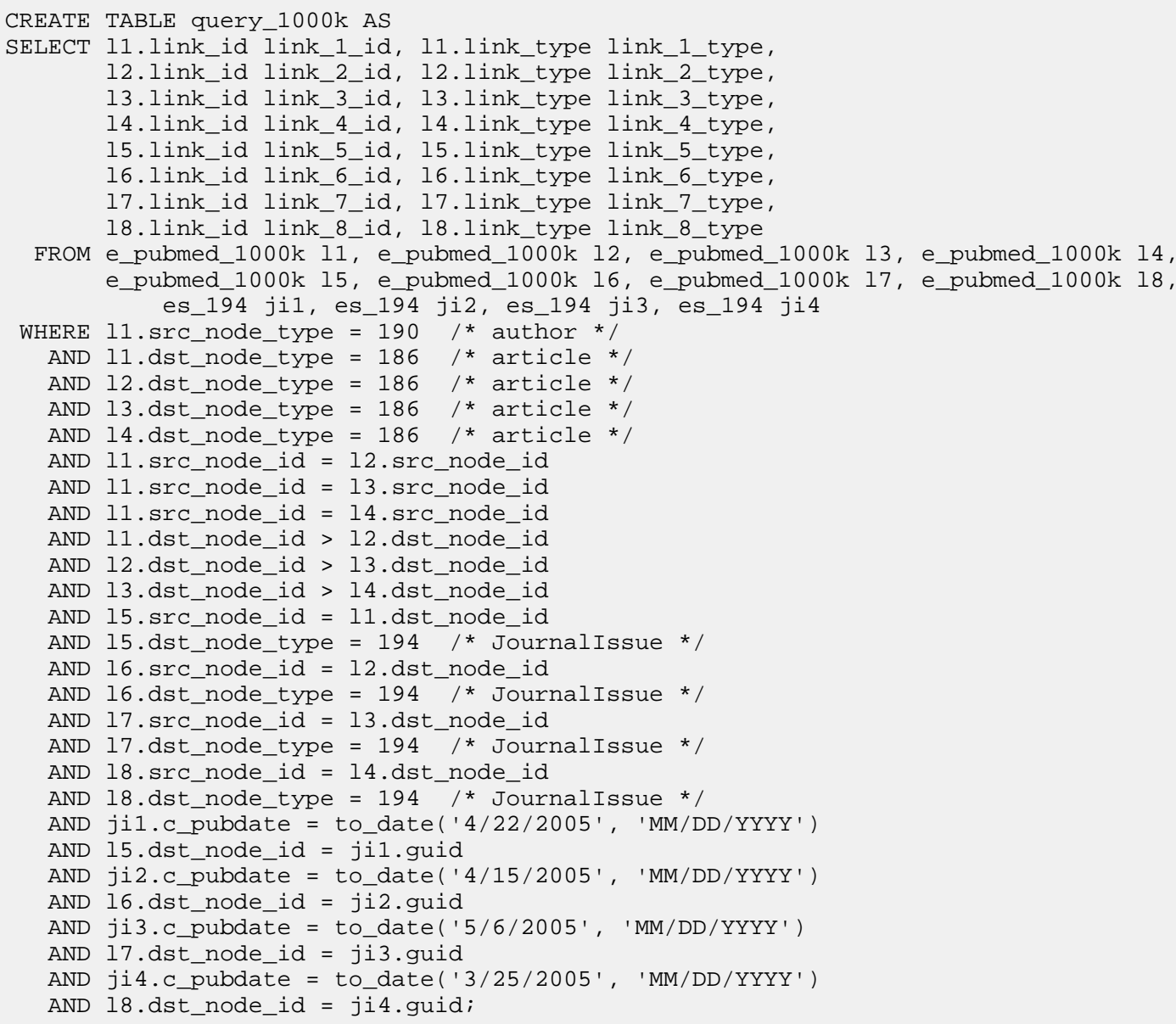

\begin{tabular}{|c|c|}
\hline \multicolumn{1}{|c|}{ time (seconds) } & \multicolumn{1}{c|}{ rows } \\
\hline 8640 (2.4 hours) & 1 \\
\hline
\end{tabular}

This query takes 2.4 hours on a million vertex test graph. Graphs that would be used in real analytic problems would be orders of magnitude larger, resulting in even longer query times.

This query can also be efficiently implemented by dividing it into four paths through the pattern query, one path for each leaf condition in the pattern query tree. Each of the paths is then joined in a final step. This approach requires five SQL queries: one for each of the four paths, plus the final join. 


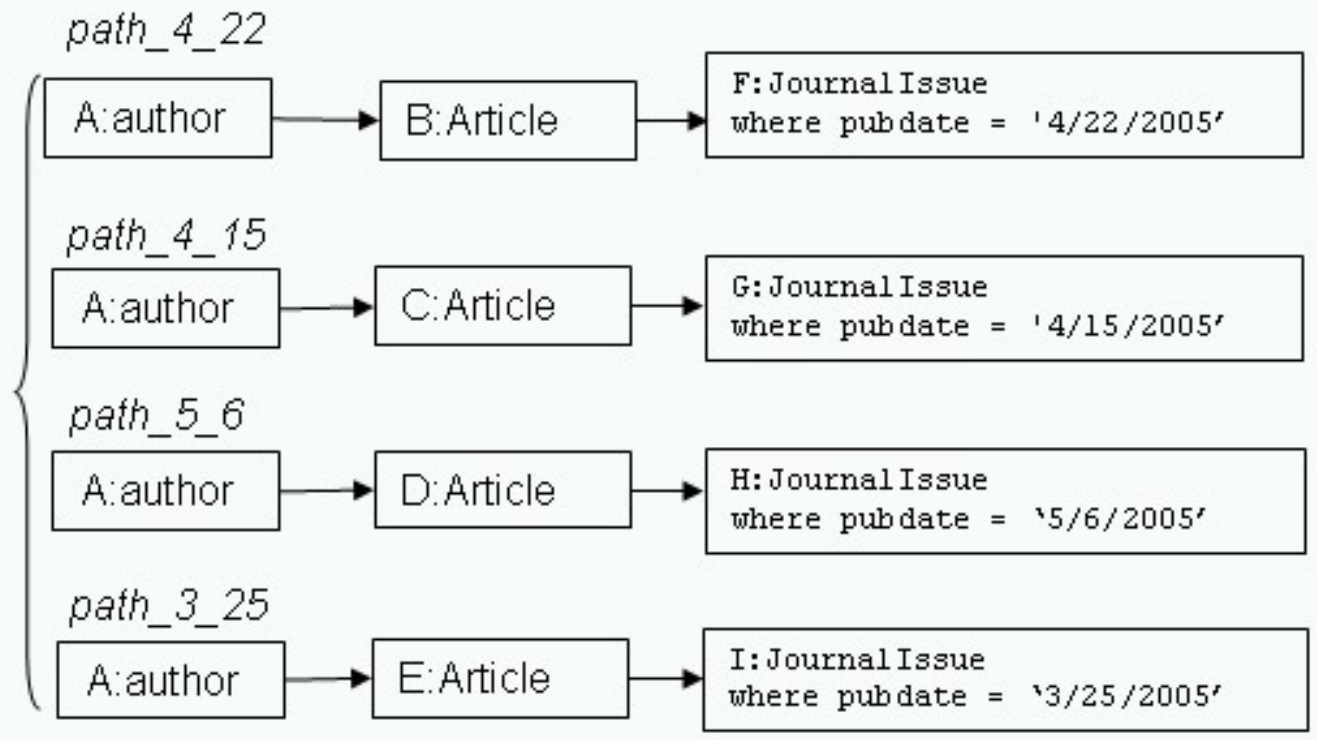

CREATE TABLE path_4_22 AS

SELECT 11.src_node_id e1_src_node_id, 11.src_node_type e1_src_node_type, 11.link_id e1_link_id, 11.link_type e1_link_type,

11.dst_node_id e1_dst_node_id, 11.dst_node_type e1_dst_node_type,

12.src_node_id e2_src_node_id, 12.src_node_type e2_src_node_type,

12.link_id e2_link_id, 12.link_type e2_link_type,

12.dst_node_id e2_dst_node_id, 12.dst_node_type e2_dst_node_type

FROM e_pubmed_1000k 11, e_pubmed_1000k 12, es_194 ji

WHERE 11.src_node_type $=190 / *$ author */

AND 11.dst_node_type $=186 /$ * article */

AND 12.src_node_id $=11 . d s t \_n o d e \_i d$

AND 12.dst_node_type $=194 / *$ Journallssue */

AND ji.c_pubdate = to_date ('4/22/2005', 'MM/DD/YYYY')

AND 12.dst_node_id $=$ ji.guid

;

CREATE TABLE path_4_15 AS

SELECT 11.src_node_id e1_src_node_id, 11.src_node_type el_src_node_type, 11.link_id e1_link_id, l1.link_type e1_link_type,

11.dst_node_id e1_dst_node_id, l1.dst_node_type e1_dst_node_type,

12.src_node_id e2_src_node_id, 12.src_node_type e2_src_node_type,

12.link_id e2_link_id, 12.link_type e2_link_type,

12.dst_node_id e2_dst_node_id, 12.dst_node_type e2_dst_node_type

FROM e_pubmed_1000k 11, e_pubmed_1000k 12, es_194 ji

WHERE 11.src_node_type $=190 /$ * author */

AND 11.dst_node_type $=186 /$ *article */

AND 12.src_node_id $=11$. dst_node_id

AND 12.dst_node_type $=194 /$ * JournalIssue */

AND ji.c_pubdate = to_date ('4/15/2005', 'MM/DD/YYYY')

AND 12.dst_node_id $=$ ji.guid

;

CREATE TABLE path_5_6 AS

SELECT 11.src_node_id e1_src_node_id, 11.src_node_type e1_src_node_type, 11.link_id e1_link_id, l1.link_type e1_link_type,

11.dst_node_id e1_dst_node_id, l1.dst_node_type e1_dst_node_type,

12.src_node_id e2_src_node_id, 12.src_node_type e2_src_node_type,

12.link_id e2_link_id, 12.link_type e2_link_type,

12.dst_node_id e2_dst_node_id, 12 .dst_node_type e2_dst_node_type

FROM e_pubmed_1000k 11, e_pubmed_1000k 12, es_194 ji

WHERE 11.src_node_type $=190 /$ * author */

AND 11.dst_node_type $=186 /$ * article */

AND 12.src_node_id $=11$. dst_node_id

AND 12.dst_node_type $=194 /$ * JournalIssue */ 
AND ji.c_pubdate = to_date ('5/6/2005', 'MM/DD/YYYY')

AND 12.dst_node_id $=$ ji.guid

;

CREATE TABLE path_3_25 AS

SELECT 11.src_node_id e1_src_node_id, 11.src_node_type e1_src_node_type,

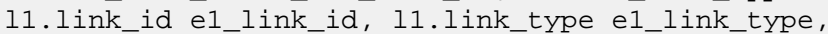

11.dst_node_id e1_dst_node_id, 11.dst_node_type e1_dst_node_type,

12.src_node_id e2_src_node_id, 12.src_node_type e2_src_node_type,

12.link_id e2_link_id, 12.link_type e2_link_type,

12.dst_node_id e2_dst_node_id, 12.dst_node_type e2_dst_node_type

FROM e_pubmed_1000k 11, e_pubmed_1000k 12, es_194 ji

WHERE 11.src_node_type $=190 / *$ author $* /$

AND 11.dst_node_type $=186 / *$ article */

AND 12.src_node_id $=11 . d s t \_n o d e \_i d$

AND 12.dst_node_type $=194 / *$ JournalIssue * /

AND ji.c_pubdate = to_date ('3/25/2005', 'MM/DD/YYYY')

AND 12.dst_node_id = ji.guid

;

CREATE TABLE query_result AS

SELECT p1.e1_link_id link_1_id, p1.e1_link_type link_1_type, p1.e2_link_id, link_5_id

p1.e2_link_type link_5_type, p2.ê1_link_id link_2_id, p2.e1_link_type link_2_type, p2.e2_link_id, link_6_id

p2.e2_link_type link_6_type,

p3.e1_link_id link_3_id, p3.e1_link_type link_3_type, p3.e2_link_id, link_7_id

p3.e2_link_type link_7_type,

p4.el_link_id link_4_id, p4.e1_link_type link_4_type, p4.e2_link_id, link_8_id

p4.e2_link_type link_8_type

FROM path_4_22 p1, path_4_15 p2, path_5_6 p3, path_3_25 p4

WHERE p1.e1_src_node_id $=$ p2.e1_src_node_id /* make sure that the author vertices are the same $\star /$

AND p2.e1_src_node_id $=$ p3.e1_src_node_id

AND p3.e1_src_node_id $=$ p4.e1_src_node_id

AND p1.e1_dst_node_id > p2.e1_dst_node_id /* order the article vertices */

AND p2.e1_dst_node_id > p3.e1_dst_node_id

AND p3.e1_dst_node_id > p4.e1_dst_node_id

\begin{tabular}{|c|c|c|}
\hline & time (seconds) & rows \\
\hline path_4_22 & 0.344 & 1559 \\
\hline path_4_15 & 1.0 & 1388 \\
\hline path_5_6 & 0.828 & 1660 \\
\hline path_3_25 & 0.467 & 493 \\
\hline join the paths & 0.280 & 1 \\
\hline total & $\sim 3.0$ & \\
\hline
\end{tabular}

\section{Pattern Queries Constrained by Vertex Type}

As discussed above, pattern queries that are constrained only by the types of the pattern vertices do combinatoric pattern matching. In this section we give a few examples of the how these queries can be translated into single SQL statements. The performance of these single SQL statements is very poor. By generating SQL for paths through the pattern and then joining these paths, query performance can be dramatically improved.

\section{Query 1}


Find subgraphs that consist of an author that has published two articles in the same journal.
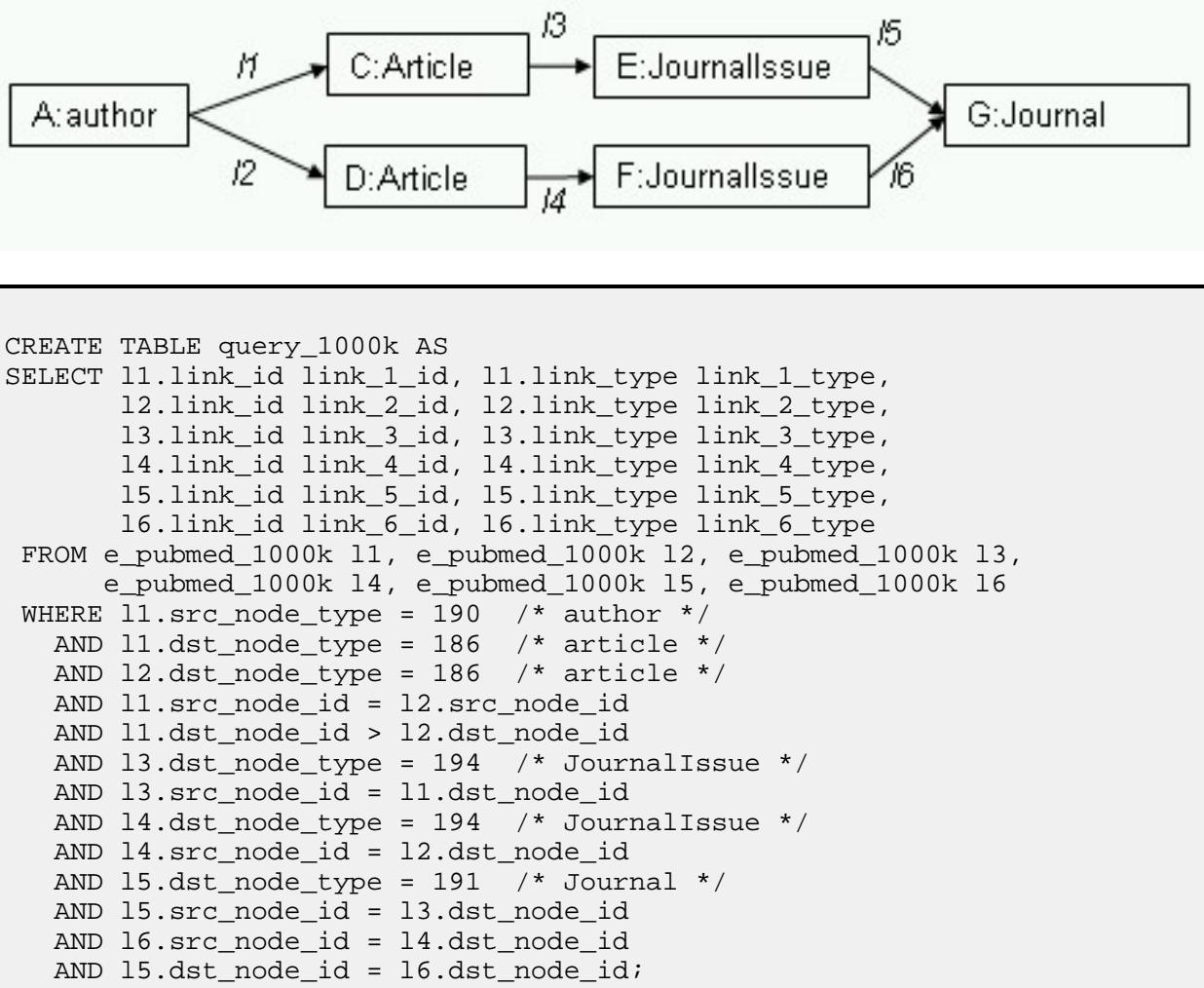

\begin{tabular}{|c|c|}
\hline time (seconds) & rows \\
\hline 54000 (15 hours) & 39036649 \\
\hline
\end{tabular}

The subgraphs that match Query 1 can be found much more rapidly using two sub-queries. The first query finds a linear path through the pattern (author --> article --> JournalIssue --> journal). The second query performs a self-join on the result of this query. This self-join finds the paths that share a common author and journal, but have different articles. The join also orders the article vertices so that the resulting subgraphs are unique.

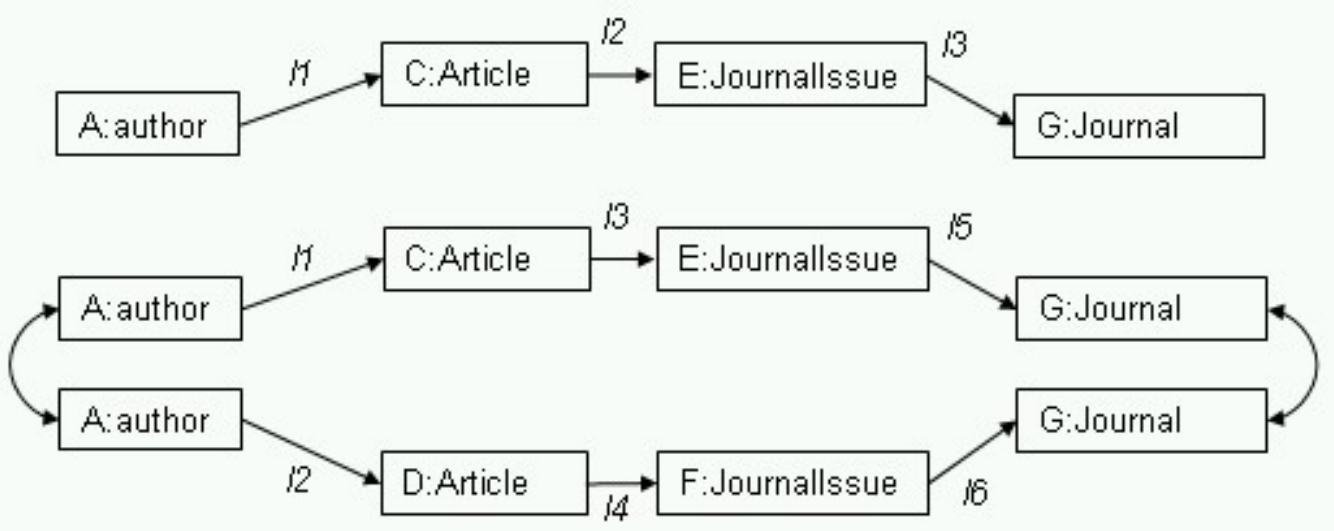


CREATE TABLE query_half AS

SELECT 11.src_node_id e1_src_node_id, 11.src_node_type e1_src_node_type, 11.1ink_id el_link_id, 11.link_type el_link_type,

11.dst_node_id e1_dst_node_id, 11.dst_node_type e1_dst_node_type,

12.src_node_id e2_src_node_id, 12.src_node_type e2_src_node_type,

12.link_id e2_link_id, 12.link_type e2_link_type,

12.dst_node_id e2_dst_node_id, 12.dst_node_type e2_dst_node_type,

13.src_node_id e3_src_node_id, 13.src_node_type e3_src_node_type,

13. link_id e3_link_id, 13. link_type e3_link_type,

13.dst_node_id e3_dst_node_id, 13.dst_node_type e3_dst_node_type

FROM e_pubmed_1000k 11, e_pubmed_1000k 12, e_pubmed_1000k 13

WHERE I1.src_node_type $=190 / *$ author */

AND 11.dst node type $=186 / *$ article */

AND 12.src_node_id $=11 . d s t \_n o d e \_i d$

AND 12.dst_node_type $=194 /$ * Journalissue $* /$

AND 13.src node id $=12$.dst node id

AND 13.dst_node_type $=191 /$ * Journal * $/$

;

CREATE TABLE query_1000k AS

SELECT h1.e1_link_id, h1.e1_link_type,

h1.e2 link id e3 link id, h1.e2 link type e3 link type,

h1.e3_link_id e5_link_id, h1.e3_link_type e5_link_type,

h2.e1_link_id e2_link_id, h2.e1_link_type e2_link_type,

h2.e2_link_id e4_link_id, h2.e2_link_type e4_link_type,

h2.e3_link_id e6_link_id, h2.e3_link_type e6_link_type

FROM query_half h1, query_half h2

WHERE h1.e1 src node id $=$ h2.e1 src node id

AND h1.e3_dst_node_id $=$ h2.e3_dst_node_id

AND h1.e1_dst_node_id > h2.e1_dst_node_id

\begin{tabular}{|c|l|l|}
\hline & \multicolumn{1}{|c|}{ time (seconds) } & rows \\
\hline find author to journal path & 114.0 & 13696874 \\
\hline join path result & $900(15$ minutes $)$ & 39036649 \\
\hline total & $1014(\sim 17$ minutes $)$ & \\
\hline
\end{tabular}

\section{Query 2}

Find subgraphs that consist of an author who has published four articles in the journal Physical Review Letters.

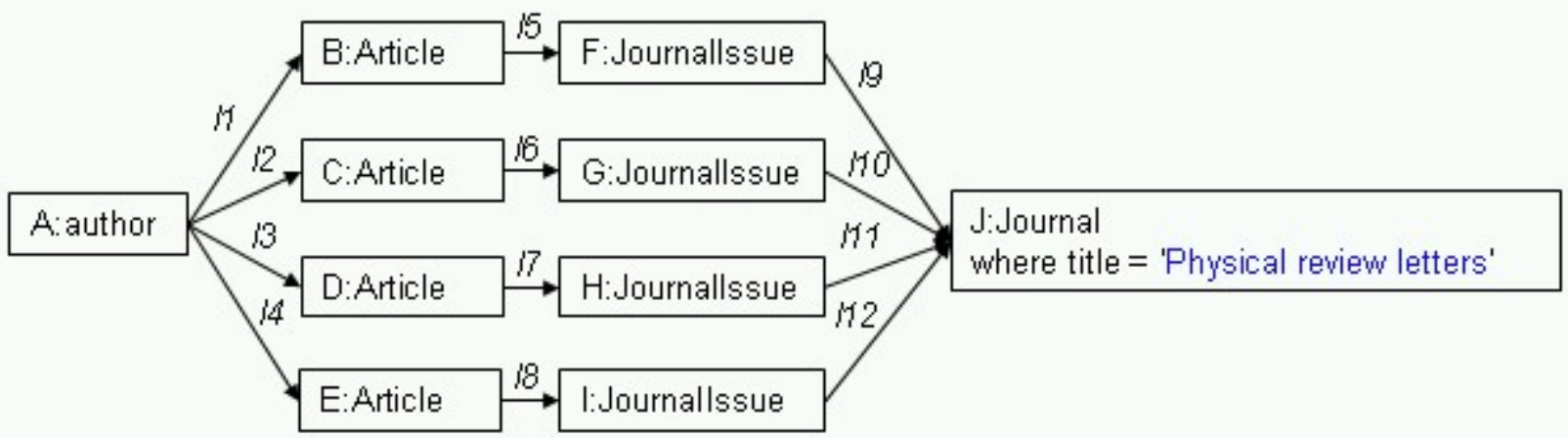

A single SQL query that implements this pattern query is shown below: 


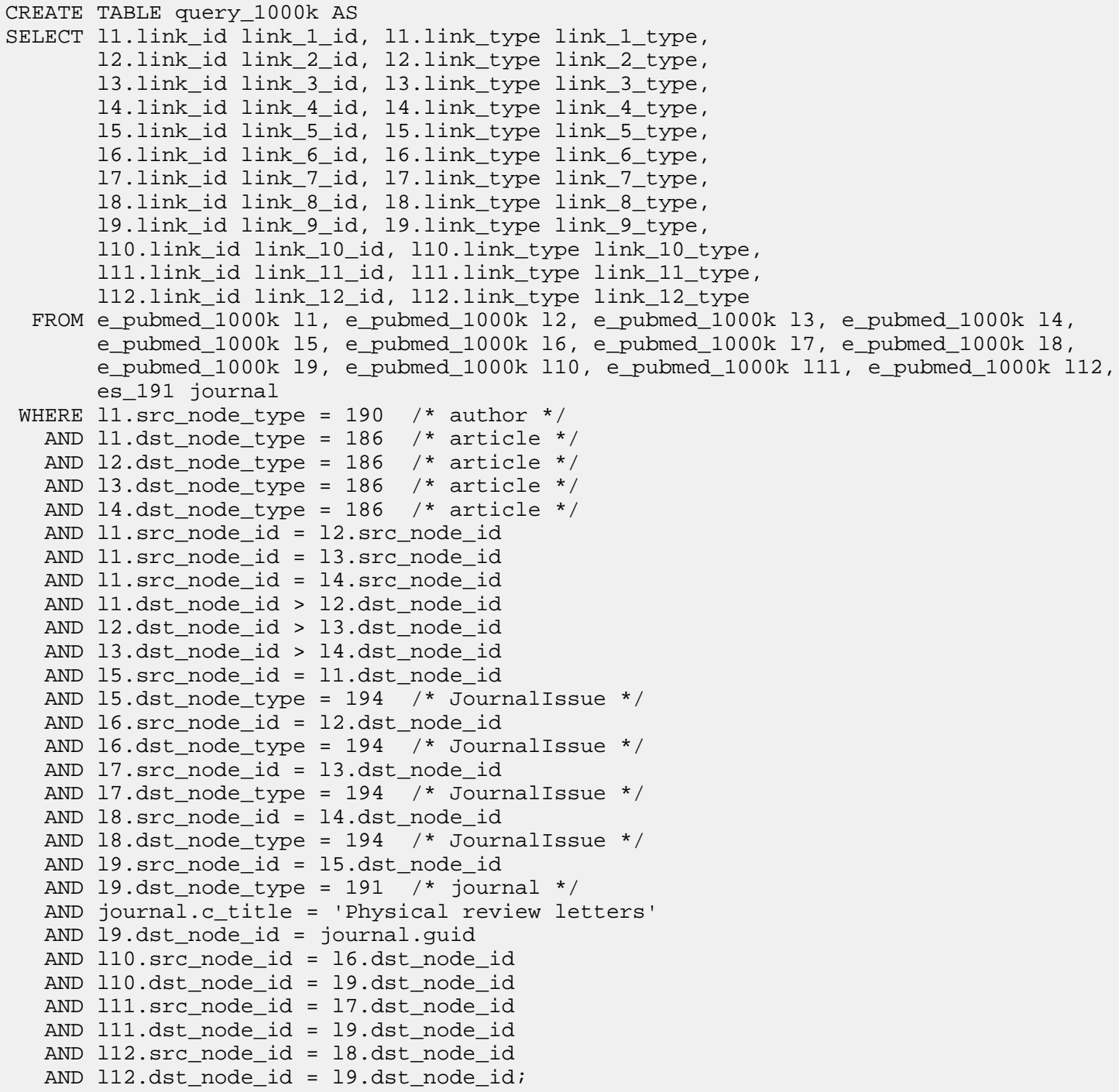

\begin{tabular}{|l|l|}
\hline \multicolumn{1}{|c|}{ time (hours) } & \multicolumn{1}{c|}{ rows } \\
\hline 16 hours & did not complete \\
\hline
\end{tabular}

If the intermediate joins used by the query planner execute the edge table self joins in a breath first order there can be large intermediate results. A depth first query strategy, where SQL is generated for paths through the pattern yields dramatically better query performance. This is shown below where the first query calculates a path through the pattern and the second query joins the paths. 

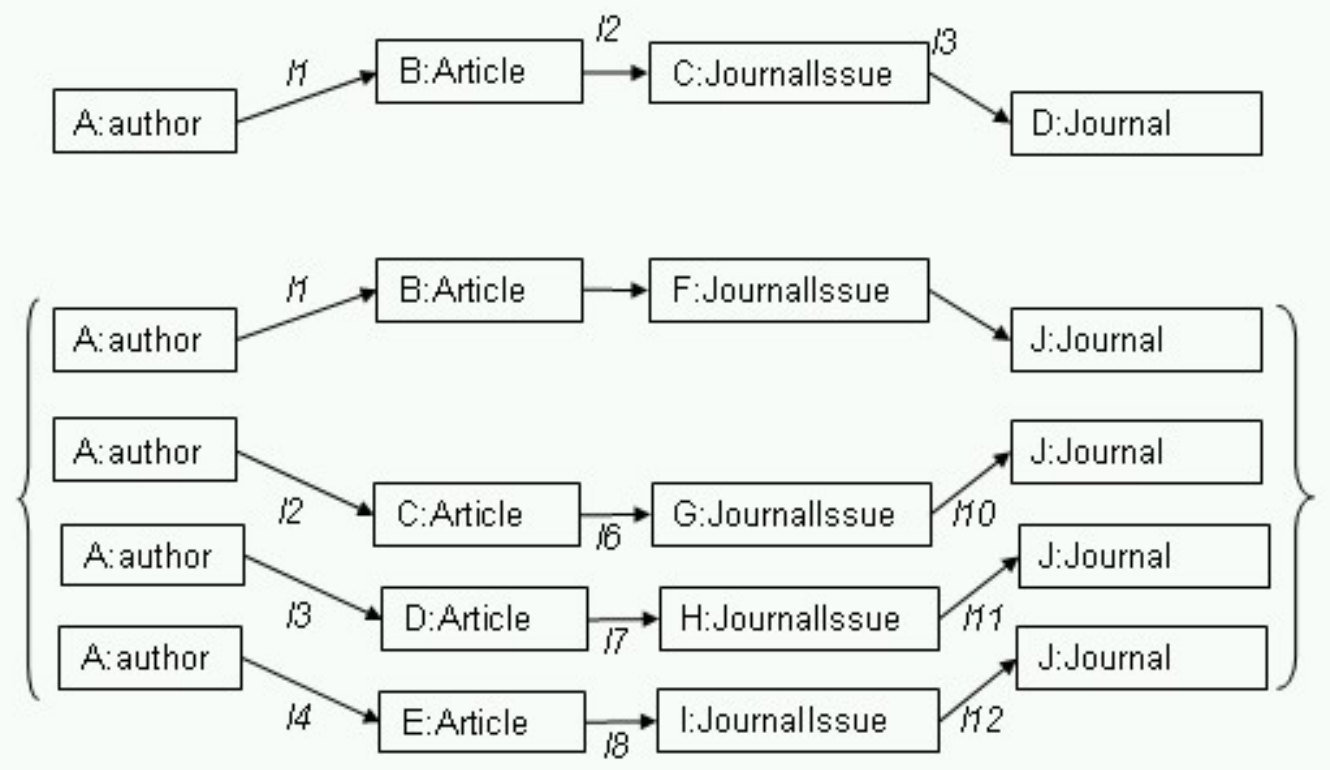

CREATE TABLE query_half AS

SELECT 11.src_node_id e1_src_node_id, 11.src_node_type e1_src_node_type, 11.link_id e1_link_id, 11.link_type e1_link_type,

11.dst_node_id e1_dst_node_id, 11.dst_node_type e1_dst_node_type, 12.src_node_id e2_src_node_id, 12.src_node_type e2_src_node_type, 12.link_id e2_link_id, $12 . \overline{l i n k}$ _type é2_link_type,

12.dst_node_id e2_dst_node_id, 12.dst_node_type e2_dst_node_type,

13.src_node_id e3_src_node_id, 13.src_node_type e3_src_node_type,

13.link_id e3_link_id, 13.link_type e3_link_type,

13.dst node id e3 dst node id, 13.dst node type e3 dst node type

FROM e_pubmed_1000k 11, e_pubmed_1000k 12, e_pubmed_1000k 13,

(select guid from es_191 where c_title = 'Physical review letters') journal

WHERE 11.src node type $=190 / *$ author */

AND 11.dst_node_type $=186 / *$ article $* /$

AND 12.src_node_id $=11$. dst_node_id

AND 12.dst node type $=194 / *$ Journalissue *

AND 13.src_node_id $=12$. dst_node_id

AND 13.dst_node_type $=191 /$ * Journal */

AND 13.dst_node_id = journal.guid

CREATE TABLE query_1000k AS

SELECT h1.e1_link_id,

h1.e2_link_type e5_link_type,

h1.e3 link id e9 link id, h1.e3 link type e9 link type,

h2.e1_link_id e2_link_id, h2.e1_link_type e2_link_type,

h2.e2_link_id e6_link_id, h2.e2_link_type e6_link_type,

h2.e3 link_id e10_link_id, h2.e3_link_type e10_link_type,

h3.e1_link_id e3_link_id, h3.e1_link_type e3_link_type,

h3.e2_link_id e7_link_id, h3.e2_link_type e7_link_type,

h3.e3_link_id e11_link_id, h3.e3_link_type e11_link_type,

h4.e1_link_id e4_link_id, h4.e1_link_type e4_link_type,

h4.e2_link_id e8_link_id, h4.e2_link_type e8_link_type,

h4.e3_link_id e12_link_id, h4.e3_link_type e12_link_type

FROM query_half h1, query_half h2, query_half h3, query_half h4

WHERE h1.e1_src_node_id $=$ h2.e1_src_node_id / t the source vertices at the start of the

pattern are the same */

AND h2.e1_src_node_id $=$ h3.e1_src_node_id

AND h3.e1_src_node_id $=$ h4 .e1_src_node_id

AND h1.e3_dst_node_id = h2.e3_dst_node_id /* the dest. vertices at the end of the pattern

are the same */

AND h2.e3_dst_node_id $=$ h3.e3_dst_node_id

AND h3.e3_dst_node_id $=$ h4.e3_dst_node_id

AND h1.e1_dst_node_id > h2.e1_dst_node_id /* order the article vertices */ 
AND h2.e1_dst_node_id > h3.e1_dst_node_id

AND h3.e1_dst_node_id > h4.e1_dst_node_id

;

\begin{tabular}{|c|l|l|}
\hline & \multicolumn{1}{|c|}{ time (seconds) } & rows \\
\hline path query & 1.8 & 27418 \\
\hline part 2 & 20.0 & 957272 \\
\hline total & 21.8 & \\
\hline
\end{tabular}

\section{Query 3}

Find the subgraphs that consist of two authors who have co-authored two papers.

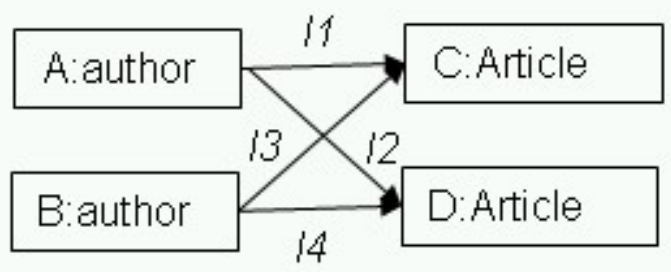

A single SQL statement that implements this query is shown below:

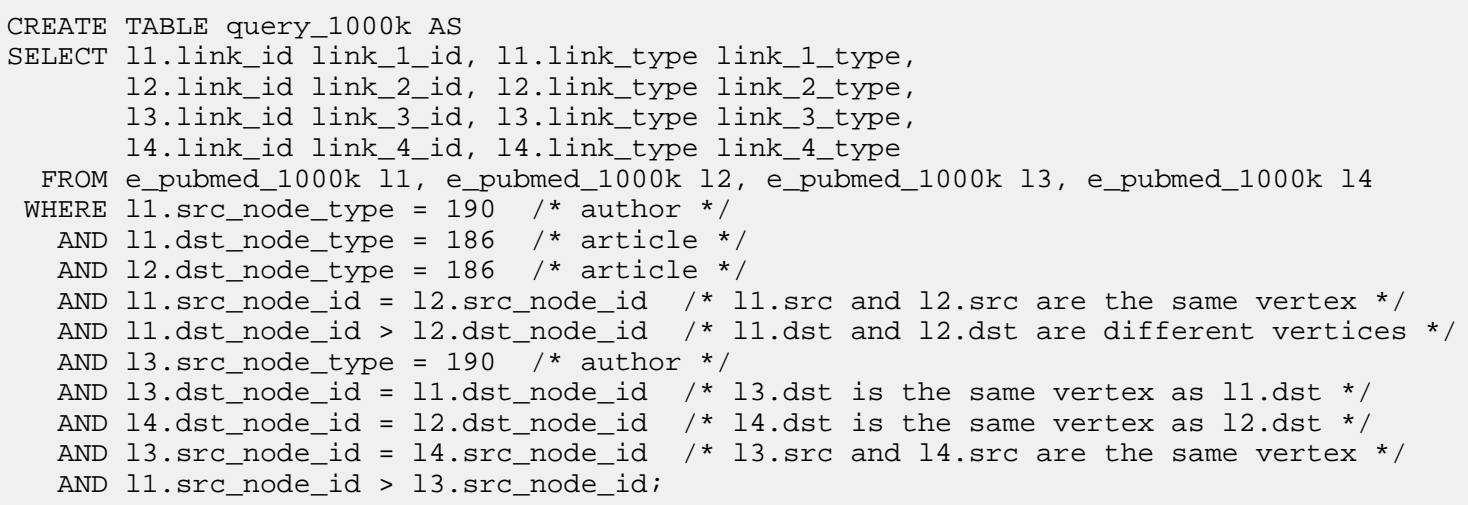

\begin{tabular}{|c|c|}
\hline time (seconds) & \multicolumn{1}{c|}{ rows } \\
\hline 3480.0 (58 minutes) & 19067116 \\
\hline
\end{tabular}

This query can be calculated more efficiently by breaking it into two sub-queries and then joining the result, as shown in the diagram below. 

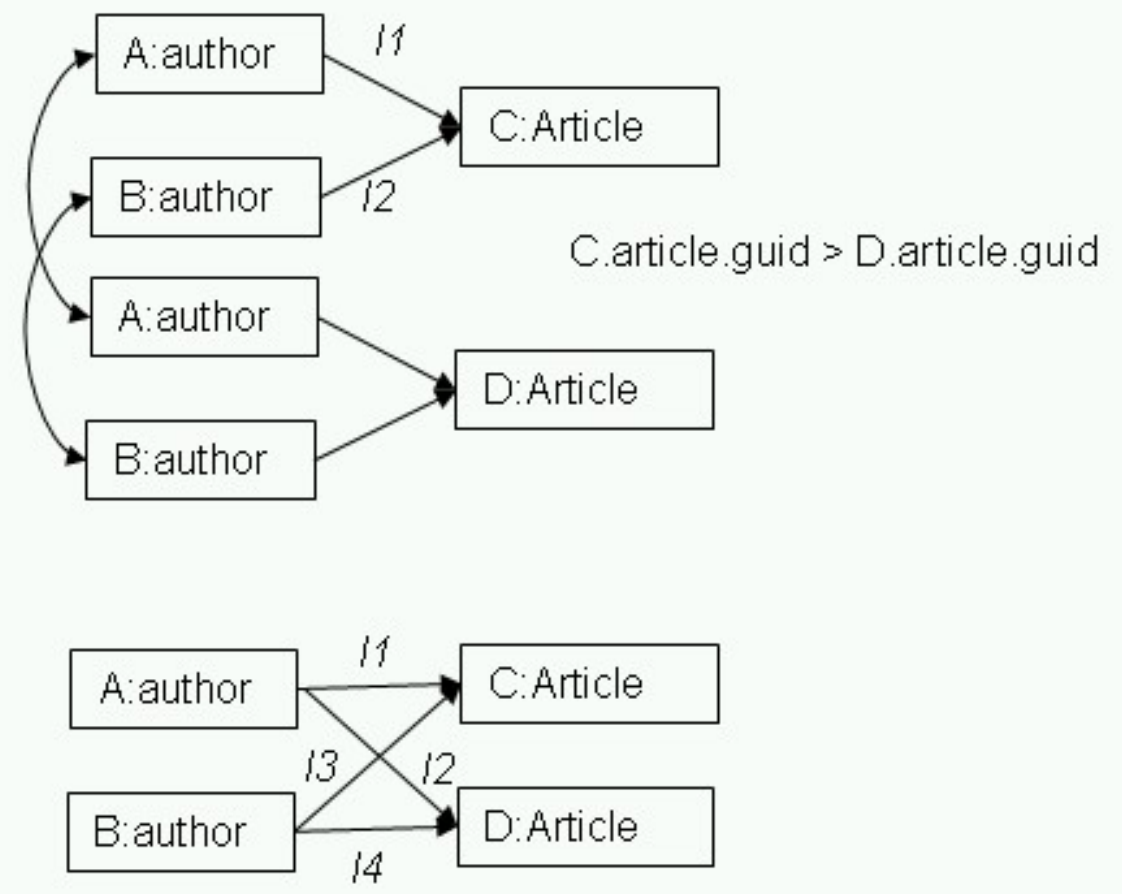

CREATE TABLE query_half AS

SELECT 11.src node id e1_src_node_id, 11.src_node_type e1_src_node_type, 11.1ink_id e1_link_id,

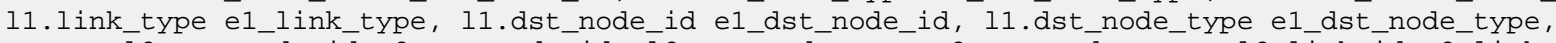
12.src_node_id e2_src_node_id, 12.src_node_type e2_src_node_type, 12.1ink_id e2_link_id, 12.1ink_type e2_link_type, 12.dst_node_id e2_dst_node_id, 12.dst_node_type e2_dst_node_type FROM e_pubmed_1000k 11, e_pubmed_1000k 12

WHERE 11.src_node_type $=190 / *$ author $* /$

AND 11.dst node type $=186 / *$ article */

AND 12.src_node_type $=190 / *$ author */

AND 11.dst_node_id $=12 . d s t \_n o d e \_i d$

AND 11.src_node_id > 12.src_node_id;

CREATE TABLE query_result AS

SELECT h1.e1_link_id e1_link_id, h1.e1_link_type e1_link_type, h1.e2_link_id e3_link_id, h1.e2_link_type e3_link_type, h2.e1_link_id e2_link_id, h2.e1_link_type e2_link_type, h2.e2_link_id e4_link_id, h2.e2_link_type e4_link_type

FROM query_half h1, query_half h2

WHERE h1.e1_src_node_id $=$ h2.e1_src_node_id

AND $h 1 . e 2$ src node id $=h 2 . e 2$ src node id

AND h1.e1_src_node_id > h2.e2_src_node_id

AND h1.e1_dst_node_id > h2.e1_dst_node_id

\begin{tabular}{|l|l|l|}
\hline & \multicolumn{1}{|c|}{ time (seconds) } & 7297174 \\
\hline part 1 & 51.0 & 19067116 \\
\hline part 2 & 336.0 & \\
\hline total & $387(6.45$ minutes $)$ & \\
\hline
\end{tabular}

\section{Query 4}

In knowledge discovery applications it can be important to be able to specify a query where an edge is excluded from the pattern. The query below specifies a pattern that contains an article that has an 
associated grant and an article that does not have an associated grant. A single SQL statement for this query is shown below.

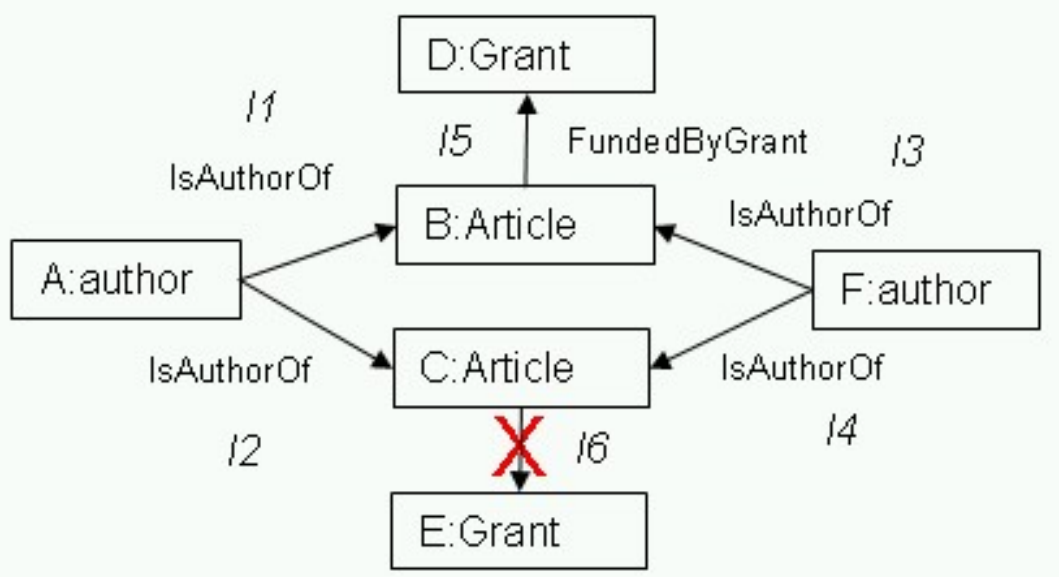

$\begin{array}{ll}\text { CREATE } & \text { TABLE query_1000k AS } \\ \text { SELECT } & \text { 11.link_id link_1_id, 11.link_type link_1_type, } \\ & \text { 12.link_id link_2_id, 12.link_type link_2_type, } \\ & \text { 13.link_id link_3_id, 13.link_type link_3_type, } \\ & \text { 14.link_id link_4_id, 14.link_type link_4_type, } \\ & \text { 15.link_id link_5_id, 15.link_type link_5_type }\end{array}$

FROM e_pubmed_1000k 11, e_pubmed_1000k 12, e_pubmed_1000k 13, e_pubmed_1000k 14, e_pubmed_1000k 15

WHERE 11.link_type $=313 \quad / *$ IsAuthorof */

AND 11.src_node_type $=190 / *$ author */

AND 11.dst_node_type $=186 / *$ article */

AND 12.link_type $=313 \quad /$ IsAuthorof $* /$

AND 12.dst_node_type $=186 /$ * article */

AND 11.src node id $=12 . \mathrm{src}$ node id /* $11 . \mathrm{src}$ and $12 . \mathrm{src}$ are the same vertex */

AND 11.dst_node_id > 12.dst_node_id /* l1.dst and 12.dst are different vertices */

AND 15.link_type $=312 \quad / *$ FundedByGrant */

AND 15.src_node_id $=11$. dst_node_id

AND 15.dst_node_type $=185 /$ * Grant */

AND 13.link_type $=313 \quad / *$ IsAuthorof $* /$

AND 13.src_node_type $=190 / *$ author */

AND 13.dst_node_id $=11 . d s t \_n o d e \_i d / * 13 . d s t$ is the same vertex as $11 . d s t * /$

AND 14.1ink_type $=313 \quad /$ IsAuthorof $* /$

AND 14.dst_node_id $=12 . d s t \_n o d e \_i d ~ / * 14 . d s t$ is the same vertex as $12 . d s t * /$

AND 13.src_node_id $=14 . \mathrm{src}$ _node_id $/ * 13 . \mathrm{src}$ and $14 . \mathrm{src}$ are the same vertex $* /$

AND 11.src_node_id > 13.src_node_id

AND NOT EXISTS (

SELECT 1

FROM e_pubmed_1000k 16

WHERE 16.link_type $=312$

AND 16.src_node_id $=14$. dst_node_id

/* FundedByGrant */

)

AND 16.dst_node_type $=185 / *$ grant */

\begin{tabular}{|l|l|}
\hline \multicolumn{1}{|c|}{ time (seconds) } & rows \\
\hline $6840.0(1.9$ hours $)$ & 7630 \\
\hline
\end{tabular}

Calculating paths through the query yields considerably better performance: 


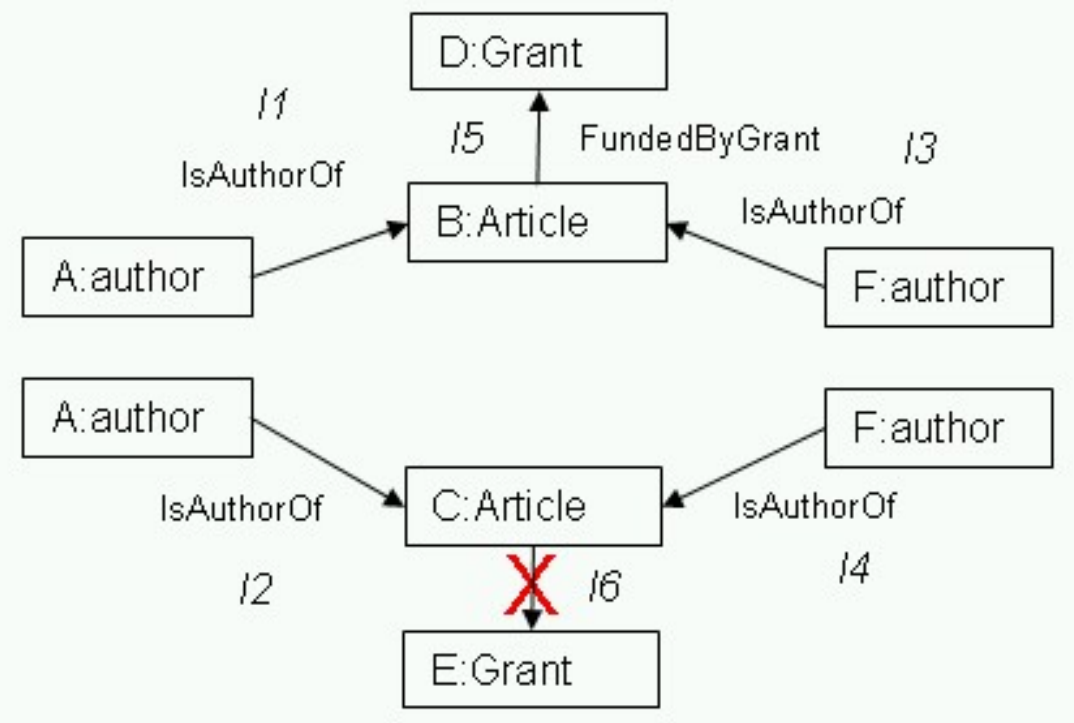

CREATE TABLE query_half_grant AS

SELECT 11.src_node_id e1_src_node_id, 11.src_node_type e1_src_node_type, 11.link_id e1_link_id, 11.1ink_type e1_link_type, 11.dst_node_id e1_dst_node_id, 11.dst_node_type e1_dst_node_type, 13.src_node_id e3_src_node_id, 13.src_node_type e3_src_node_type, 13.1ink_id e3_link_id, 13.link_type e3_link_type, 13.dst_node_id e3_dst_node_id, 13.dst_node_type e3_dst_node_type, 15.src_node_id e5_src_node_id, 15.src_node_type e5_src_node_type, 15.1ink_id e5_link_id, 15.link_type e5_link_type, 15.dst_node_id e5_dst_node_id, $15 . \mathbf{d s t}$ _node_type e5_dst_node_type, FROM e pubmed $1000 \mathrm{k} 11$, e pubmed_1000k 13, e pubmed 1000k 15

WHERE 11.src_node_type $=190 / *$ author $* /$

AND 11.1ink_type $=313 \quad / *$ IsAuthorof */

AND 11.dst node type $=186 / *$ article */

AND 13.src_node_type $=190 / *$ author */

AND 13.1ink_type $=313 /$ * IsAuthorof */

AND 11.dst node id $=13$. dst node id

AND 11.src_node_id > 13.src_node_id

AND 15.src_node_type $=186 /$ * article */

AND 15.src node id $=13$. dst node id

AND 15.dst_node_type $=185 /$ * grant */

;

CREATE TABLE query_half_no_grant AS

SELECT 12.src_node_id e2_src_node_id, 12.src_node_type e2_src_node_type, 12.link_id e2_link_id, 12.1ink_type e2_link_type, 12.dst_node_id e2_dst_node_id, 12.dst_node_type e2_dst_node_type, 14.src_node_id e4_src_node_id, I4.src_node_type e4_src_node_type, 14.1ink_id e4_link_id, 14.link_type e4_link_type, 14.dst_node_id e4_dst_node_id, 14. dst_node_type e4_dst_node_type FROM e_pubmed_1000k 12, e_pubmed_1000k 14

WHERE 12.src_node_type $=190 /$ author $* /$

AND 12.1ink_type $=313 /$ * IsAuthorof */

AND 12.dst node type $=186 / *$ article */

AND 14.src_node_type $=190 / *$ author $* /$

AND 14.1ink_type $=313 /$ * IsAuthorof */

AND 12.dst node id $=14$. dst_node_id

AND 12.src_node_id > 14.src_node_id

AND NOT EXISTS (

SELECT 1

FROM e_pubmed_1000k 16

WHERE 16.1 ink_type $=312$

AND 16.src node id $=14$. dst node id

/* FundedByGrant */

AND 16.dst_node_type $=185 /$ * grant */

CREATE TABLE query_result AS

SELECT h1.e1_link_id, h1.e1_link_type, h1.e3_link_id, h1.e3_link_type, h1.e5_link_id, 


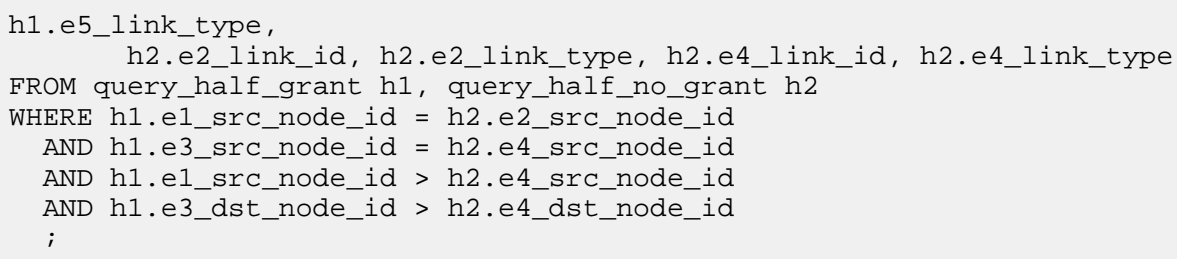

\begin{tabular}{|c|l|l|}
\hline & \multicolumn{1}{|c|}{ time (seconds) } & \multicolumn{1}{c|}{ rows } \\
\hline part 1 (query_half_grant) & 162.0 & 548855 \\
\hline part 2 (query_half_no_grant) & 78.0 & 7085770 \\
\hline join & 42 & 7630 \\
\hline total & $282.0(4.7$ minutes) & \\
\cline { 1 - 2 } & &
\end{tabular}

\section{Acknowledgments}

Justin Levandoski did some of the early foundation work on translating pattern queries into single SQL statements and benchmarking these queries on multiple databases.

\section{References}

1. SQL 1999: Understanding Relational Language Components by Jim Melton and Alan R. Simon, Morgan Kaufmann, 2002

2. A visual lanquage for querying and updating graphs by $\mathrm{H}$. Blau, N. Immerman and D. Jensen, 2002 , University of Massachusetts Amherst Computer Science Technical Report 2002-037

3. Proximity 4.2 QGraph Guide by David Jensen, 2006, University of Massachusetts Amherst

4. The Graph Query Language, David Silberberg, July 18, 2006, from a presentation at the Lawrence Livermore National Laboratory

5. A Semantic Graph Query Lanquage by Kaplan, I.L., October 16, 2006, Lawrence Livermore National Laboratory Technical Report UCRL-TR-225447

6. An Algorithm for Subgraph Isomorphism by Ullman, J, Journal of the ACM, January 1976 (Volume 23, Issue 1 , Pgs $31-42$ ) 